\title{
Medical Technology: Figuring in the Equity Factor
}

The theme of this issue of MEDICC Review-Medical Technology and Health Equity-mirrors that of the previous oneMedical Education for Health Equity. This is no coincidence. As Charles Boelen's Viewpoint in the earlier issue suggests, socially accountable medical training programs are essential to achieving health equity but are not enough; it takes a whole health system built around a shared commitment to providing quality care for all. This implies integrating people and tools, with policies that encourage communication and knowledge sharing among researchers, practitioners and the public at large and that facilitate technology transfer across borders and institutions.

What's more, as a recent article in the Bulletin of the World Health Organization points out, "health technologies are always applied in a social context."[1] Unfortunately, in many social contexts around the world, even basic medical technologies, such as laboratories equipped to do routine tests for common illnesses, are accessible only to those who live near such services and/or who can afford to pay the fees either in public or higher-priced private clinics.

Limited resources for investment are a way of life for most of the world's health systems. How, then, to use and develop technology to ease inequities rather than exacerbate them? How to work out the ethical dilemma posed by cost-benefit analysis applied to new diagnostic and therapeutic tools? How to decide who benefits from science? And how do Cuban health and governmental authorities approach these issues?

Like other developing countries, Cuba's finances are limited and often quite scarce, aggravated by restrictions on imports and trade due to the longstanding US embargo. Yet, in the Cuban policy arena, this has fine-tuned attention to priorities for research and development, and led to emphasis on training of human resources for health.

The Cuban context presents certain undoubted pluses, among these a universal public health system. This has facilitated epidemiological data collection, disease surveillance, and study of trends in population health (Tuberculosis Mortality Trends in Cuba, 1998 to 2007) that help determine priorities for diagnostic equipment, surgical devices, and treatment programs; standardization of protocols for national screening programs (A 25Year Review of Cuba's Screening Program for Early Detection of Hearing Loss); and, ultimately, strategies for distributing and using limited technological resources with maximum impact ( $A$ Comparison of Beating Heart and Arrested Heart Techniques for Mitral Valve Replacement Surgery).

The country's strong primary health care system has provided the basis for small- and large-scale studies to pinpoint where technology can resolve particular needs, as well as the clinical foundation for use in broad community-based health programs that bring technology closer to patients (Technology Transfer from Havana Hospitals to Primary Care).
A third advantage is the government's commitment to scientific research and innovation applied to public health, evidenced by the scientific research institutes that make up the Western Havana Scientific Pole, including the Cuban Neuroscience Center (CNEURO), the National Medical Genetics Center (Cuba's National Medical Genetics Program), the International Neurological Restoration Center (CIREN) (A Neurofunctional Evaluation Strategy for Presurgical Selection of Temporal Lobe Epilepsy Patients), and the Immunoassay Center. The R\&D agendas of these institutions are closely linked to the priorities of the public health system.

\section{The Cuban approach rests on two pillars: innovation and a sort of "technology triage"}

However weighty these advantages, the tough questions remain: what kind of technology to develop and how to use it best.

The Cuban approach rests on two pillars. The first is innovation in the development of domestically designed and produced technology, economically more feasible and deemed more truly appropriate (Generating Appropriate Technologies for Health Equity). Such innovation is also equity-driven, in the sense that lower costs per unit of technology extend its benefit to greater numbers of people.

The second pillar is a sort of "technology triage" in which simpler and more economical diagnostic tools are used in a first stage of broad population-based active screening, and selectively more complex and costly technology is reserved in later stages for those patients detected (Stratified Active Screening: Where Neurotechnology Meets Public Health).

In addition to the discussion of medical technology and health equity found in the following pages, we would like to draw your attention to the Global Forum for Health Research meeting in Havana, slated for November 2009 (see inside back cover), where participants will present their findings and no doubt glean a better first-hand look at the Cuban experience.

Finally, we would like to take this opportunity to second the Viewpoint of Dr. Francisco Rojas Ochoa (Cuban Health Professionals: Will Publishing Perish?), by calling on Cuban medical researchers and practitioners to make publication of their results a higher priority, in both national and international journals. They and the global health community will certainly benefit. $-\mathrm{p}$ -

\section{The Editors}

1. Saarni IS, Hofmann B, Lampe K, Lühmann D, Mäkelä M, Velasco-Garrido $\mathrm{M}$, et al. Ethical analysis to improve decision-making on health technologies. Bulletin of the World Health Organization. 2008 Aug;86(8),617-23. 